\title{
Study on the Relationship between Psychological Stress and Doctor-Patient Relationship of Cancer Patients and Their Families
}

\author{
Chunru Wang1, Ping Huang², Xiangling Jiang3*, You Li, Juncheng Guo ${ }^{5}$, Yijun Yang ${ }^{5}$, Min Guo ${ }^{3}$ \\ ${ }^{1}$ Qionghai People's Hospital, Qionghai, China \\ ${ }^{2}$ University of South China, Hengyang, China \\ ${ }^{3}$ Department of Psychology, Hainan General Hospital, Haikou, China \\ ${ }^{4}$ Hengyang Maternal and Child Health Hospital, Hengyang, China \\ ${ }^{5}$ Xiangya School of Medical Affiliated Haikou Hospital, Central South University, Haikou, China \\ Email: *g2002m@163.com
}

How to cite this paper: Wang, C.R., Huang, P., Jiang, X.L., Li, Y., Guo, J.C., Yang, Y.J. and Guo, M. (2019) Study on the Relationship between Psychological Stress and Doctor-Patient Relationship of Cancer Patients and Their Families. Journal of Behavioral and Brain Science, 9, 165-173.

https://doi.org/10.4236/jbbs.2019.94014

Received: March 13, 2019

Accepted: April 8, 2019

Published: April 11, 2019

Copyright (๑) 2019 by author(s) and Scientific Research Publishing Inc. This work is licensed under the Creative Commons Attribution International License (CC BY 4.0).

http://creativecommons.org/licenses/by/4.0/

\begin{abstract}
Objective: To research the relationship between psychological stress and doctor-patient relationship of cancer patients and their families. Methods: The patients were randomly divided into the intervention group and the control group, and PDRQ-15, pcl-c, SAS and SDS scales were selected as evaluation indexes, and the levels of norepinephrine and dopamine were compared between the patients diagnosed with PTSD cancer and those without PTSD. Results: 1) The total score of PCL-C, SAS, SDS, PDRQ-15 scale of the cancer patients and their families after the intervention of clinical psychological care was significantly lower than that of before intervention and the control group. 2) The correlation coefficients between PCL-C, SAS, SDS and PDRQ-15 of cancer patients and their relatives were $0.971,0.952$ and 0.939 respectively. The significant test $\mathrm{P}$ value was less than 0.05 and the difference was statistically significant. 3) The plasma levels of dopamine and norepinephrine in cancer patients under stress were significantly higher than those in cancer patients without stress $(\mathrm{P}<0.05)$, and the difference was statistically significant. Conclusion: After psychological Intervention of cancer patients and their families, post-traumatic stress disorder, anxiety, depression and doctor-patient relationship were all improved.
\end{abstract}

\section{Keywords}

Cancerous Person, Family Members Psychological Stress, Doctor-Patient 
Relationship

\section{Introduction}

Cancer is a systemic wasting disease with a long course of disease and high mortality rate and has developed to be the "first murderer" of health. The confirmed diagnosis of cancer will bring patients a series of psychological stress reactions such as anxiety, depression, fear and desperation [1] [2]. Besides, 99\% patient families feel stressful in the process of taking care of patients [3]. According to a research report, the negative effects arising from the stress of patient families included physical stress, psychological stress, social contact stress and financial press [4]. In the process of treatment, social communication activities generated between doctors and patients, namely the process of human psychological and behavioral communication, result in the generation, development and change of doctor-patient relationship, which is certainly closely related to social psychological factors including humans' cognitions, expectations, motivations, evaluations, attitudes and attributions and behaviors [5]. When the satisfaction of doctor-patient relationship is low, patients and their families would have negative emotions. If the psychological reactions of patients are too negative or patients fall into negative emotions for a long term, it would cause imbalance of hormone levels of patients' body, increase of secretion of adrenaline, and further bring about the decrease of immunity functions of their body, which not only affects the cooperation degree of cancer patients for various therapies, but also has an adverse effect on postoperative treatment and physical and psychological rehabilitation [6]. It can thus be seen that, the relationship between patients and their families and doctors and nurses plays an important role, and the physical and psychological health of patients and their families and the high satisfaction of doctor-patient relationship provide significant guarantee for the disease curability of patients [7]. Therefore, learning the psychological stress reactions of cancer patients and their families and taking effective psychological intervention measures for them can help them adjust their mental states, reduce stress reaction degree and restore confidence for life as soon as possible, which has a very crucial social meaning for maintaining the physical and psychological health of persons involved and is beneficial to build a harmonious doctor-patient relationship [8] [9].

The research applied a part of psychological scales to determine the satisfaction of doctor-patient relationship, anxiety, depression, PTSD (post-traumatic stress disorder) conditions of patients and their families before and after psychological crisis intervention, and compared the secretion volume of norepinephrine and dopamine of cancer patients with PTSD and those without PTSD to discuss the correlation between the psychological stress reactions-anxiety, depression, PTSD, and the satisfaction of doctor-patient relationship of cancer pa- 
tients and their families.

\section{Research Objects and Methods}

1) Research Objects

200 pairs of cancer patients hospitalized in Department of Radiotherapy of Qionghai City People's Hospital and their families were selected and completely and randomly divided into an intervention group and a control group, with 100 pairs in each group.

2) Inclusion Criteria

a) patients who were diagnosed as malignant tumor after pathologic, ultrasonic and imageological examinations; b) patients and their families over 18 years old; c) patients with the educational level of primary school and above; d) patients without serious physical diseases or psychosis; e) Karnofsky performance status (KPS) > 60 scores; $f$ ) it was expected that the survival time of patients was over half a year; g) patients who have known their disease conditions and agreed the research; h) except for those with other serious physical diseases; i) patients whose disease conditions were permitted and who were willing to participate in the research and can provide active cooperation.

3) Exclusion Criteria

a) patients with previous medical histories of mental diseases; b) patients with positive family histories of mental diseases; c) patients with serious audio-visual disorder that may affect their correct understanding and answers for questionnaires; d) patients who refused to cooperate after explanations of researchers.

4) Conventional treatment and nursing were implemented for the patients in the control group, with the course of treatment of 1 month.

5) Conventional treatment, nursing and psychological and behavioral intervention were implemented for the patients in the intervention group, and the psychological and behavioral intervention lasted for 1 month. Intervention contents were mainly from the cancer supportive care website of the United States (www.cancersupportivecare.com), and specific details were as follows:

a) Nutrition

Contents involved the basic principles of diet and common diet problems and countermeasures. Common problems mainly included appetite loss, nausea and vomiting, diarrhea, constipation, bradymasesis or dysphagia.

b) Sleep: contents involved common sleep problems, how to form good sleeping habits and how to take drugs.

c) Exercise: contents involved the advantages of exercise and suggestions.

d) Pain control guidance: contents involved the necessity of pain relief, common pain relief problems, pain reasons and types, recording of pain details, formation and utilization of pain control plans; types, using methods and treatment of possible side effects of analgesics; non-drug treatment of pain, mainly including basic principles and common methods.

e) Emotion management: contents involved emotional marks and influencing factors as well as functions and management of emotions. 
f) Psychological factors and diseases: contents involved the relationship between the both and responses.

g) Psychological distress and social support: contents involved the responses of distress and utilization of social support.

h) Behavior therapy: progressive muscle relaxation training (PMR). PMR is the most common relaxation training. The therapy frequency was 20 minutes per time and twice per day, and researchers supervised its execution and recorded.

6) Research tools

General information questionnaires, doctor-patient relationship scale (PDRQ-15), screening scale of post-traumatic stress disorder (PTSD) (PCL-C), self-rating anxiety scale (SAS) and self-rating depression scale (SDS).

7) The Patients

The patients who were diagnosed as cancer with PTSD and without PTSD were screened for the determination of levels of norepinephrine (NE) and dopamine (DA).

8) Statistical methods

The questionnaires were uniformly numbered after taken backed, and a database was established with EpiData3.1 software and SPSS18.0 was used for statistical data analysis.

\section{Results}

1) General conditions of research objects are shown in Table 1.

2) Scores of PCL-C, SAS, SDS and PDRQ-15 scales of cancer patients and

Table 1. Basic conditions of cancer patients.

\begin{tabular}{|c|c|c|}
\hline Scale & & $\mathrm{N}(100 \%)$ \\
\hline \multirow[t]{2}{*}{ Sex } & Male & 122 \\
\hline & Female & 78 \\
\hline \multirow[t]{4}{*}{ Educational level } & Primary school & 34 \\
\hline & Junior high school & 42 \\
\hline & Senior high school & 86 \\
\hline & University and above & 38 \\
\hline \multirow[t]{3}{*}{ Age } & $18-38$ & 54 \\
\hline & $39-58$ & 90 \\
\hline & $59-79$ & 56 \\
\hline \multirow[t]{6}{*}{ Cancer type } & Lung cancer & 48 \\
\hline & Gastrointestinal cancer & 34 \\
\hline & Breast cancer & 4 \\
\hline & Uterus cancer & 8 \\
\hline & Liver cancer & 24 \\
\hline & Others (lymph cancer, bladder cancer and melanocarcinoma etc.) & 82 \\
\hline
\end{tabular}


their families in the intervention group before and after intervention

The total scores of PCL-C, SAS, SDS and PDRQ-15 scales of cancer patients and their families in the intervention group before and after intervention were compared, and P values were respectively $0.015,0.037,0.028$ and 0.022 , all of which were lower than 0.05 , so the difference was statistically significant. Specific details are shown in Table 2.

3) Scores of PCL-C, SAS, SDS and PDRQ-15 scales of cancer patients and their families in the control group before and after treatment.

The total scores of PCL-C, SAS, SDS and PDRQ-15 scales of cancer patients and their families in the control group before and after treatment were compared, and P values were respectively $0.290,0.089,0.135$ and 0.078 , all of which were higher than 0.05 , so the difference was not statistically significant. Specific details are shown in Table 3.

4) Scores of PCL-C, SAS, SDS and PDRQ-15 scales of cancer patients and their families in the intervention group and control group

One month after treatment, the total scores of PCL-C, SAS, SDS and PDRQ-15 scales of cancer patients and their families in the intervention group and control group were compared, and $\mathrm{P}$ values were respectively $0.012,0.035$, 0.028 and 0.016 , all of which were lower than 0.05 , showing a statistically significant difference. Specific details are available in Table 4.

Table 2. Comparison of total scores of PCL-C, SAS, SDS and PDRQ-15 scales of cancer patients and their families in the intervention group before and after intervention.

\begin{tabular}{ccc}
\hline Scale & Total scores (meanstandard deviation) & P value \\
\hline PCL-C & $75.11 \pm 7.89^{\mathrm{A}}$ & $0.015^{\star}$ \\
SAS & $42.55 \pm 2.34^{\mathrm{B}}$ & \\
& $71.53 \pm 1.08^{\mathrm{A}}$ & $0.037^{\star}$ \\
& $71.53 \pm 1.08^{\mathrm{A}}$ & $0.028^{*}$ \\
SDS & $69.71 \pm 3.14^{\mathrm{A}}$ & \\
& $54 \pm 7.81^{\mathrm{B}}$ & $0.022^{*}$ \\
\hline
\end{tabular}

Table 3. Comparison of total scores of PCL-C, SAS, SDS and PDRQ-15 scales of cancer patients and their families in the control group before and after treatment $(n=100)$.

\begin{tabular}{ccc}
\hline Scale & Total scores (mean \pm standard deviation) & P value \\
\hline \multirow{2}{*}{ PCL-C } & $70.37 \pm 6.62^{\mathrm{A}}$ & 0.290 \\
& $68.07 \pm 4.88^{\mathrm{B}}$ & \\
SAS & $73.93 \pm 1.99^{\mathrm{A}}$ & 0.089 \\
& $69.88 \pm 5.59^{\mathrm{B}}$ & 0.135 \\
SDS & $65.79 \pm 9.37^{\mathrm{A}}$ & \\
& $70.28 \pm 6.53^{\mathrm{B}}$ & 0.078 \\
\hline
\end{tabular}

(A means before treatment and B means after treatment). 
5) Pearson correlation analysis of PCL-C, SAS and SDS with PDRQ-15 of cancer patients and their families

The correlation coefficients between PCL-C, SAS, SDS and PDRQ-15 of cancer patients and their families were respectively $0.971,0.952$ and $0.939, \mathrm{P}$ values in significance tests were all lower than 0.05 , with a statistically significant difference. Specific details are shown in Table 5.

6) DA and NE analysis of cancer patients with PTSD and those without PTSD

The plasma DA and NE levels of cancer patients under stress conditions were all higher than those of cancer patients not under stress conditions, and $\mathrm{P}$ values were all lower than 0.05 , so the difference was statistically significant, as shown in Figure 1.

Table 4. Comparison of total scores of PCL-C, SAS, SDS and PDRQ-15 scales of cancer patients and their families in the intervention group and control group $(n=100)$.

\begin{tabular}{ccc}
\hline Scale & Total scores (mean \pm standard deviation) & P value \\
\hline \multirow{2}{*}{ PCL-C } & $42.55 \pm 2.34^{\mathrm{A}}$ & $0.012^{\star}$ \\
& $68.07 \pm 4.88^{\mathrm{B}}$ & \\
SAS & $57.33 \pm 6.66^{\mathrm{A}}$ & $0.035^{*}$ \\
& $69.88 \pm 5.59^{\mathrm{B}}$ & $0.028^{*}$ \\
SDS & $54 \pm 7.81^{\mathrm{A}}$ & $0.016^{*}$ \\
& $70.28 \pm 6.53^{\mathrm{B}}$ & \\
PDRQ-15 & $60.67 \pm 4.19^{\mathrm{A}}$ & \\
\hline
\end{tabular}

( ${ }^{\star}$ means $\mathrm{p}<0.05$, A means intervention group and B means control group).

Table 5. Pearson correlation analysis of PCL-C, SAS and SDS with PDRQ-15 of cancer patients and their families.

\begin{tabular}{cccc}
\hline Scale & $\mathrm{N}$ & P value & Pearson correlation efficient with PDRQ-15 \\
\hline PCL-C & 100 & $0.025^{\star}$ & 0.971 \\
SAS & 100 & $0.037^{\star}$ & 0.952 \\
SDS & 100 & $0.014^{\star}$ & 0.939 \\
\hline
\end{tabular}

$\left({ }^{*}\right.$ means $\left.\mathrm{p}<0.05\right)$.
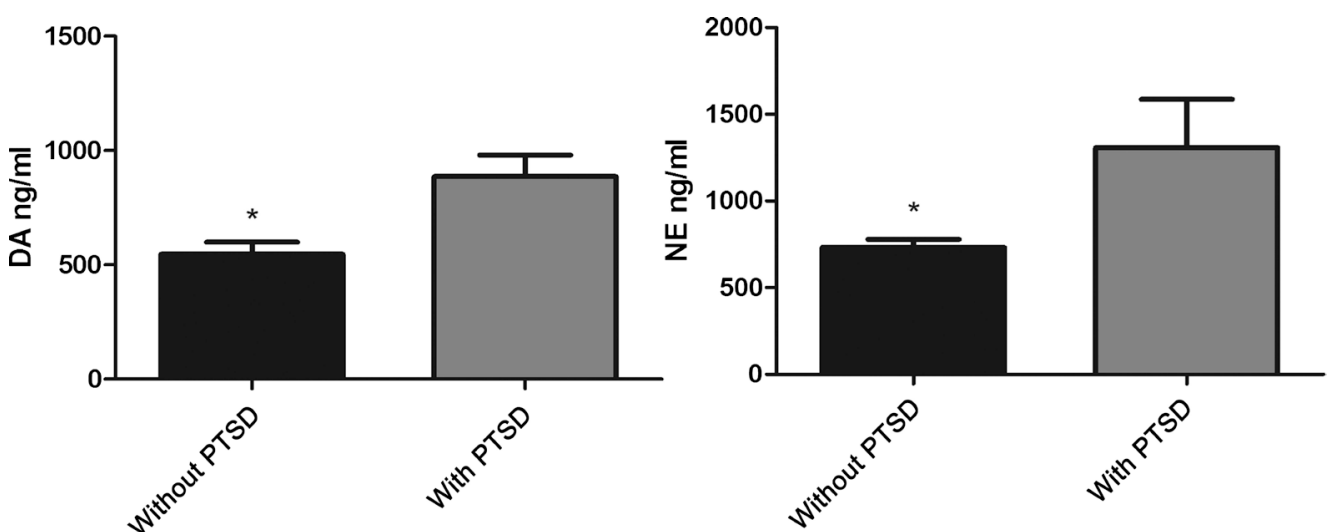

Figure 1. DA and NE levels of cancer patients with PTSD and those without PTSD. $\left({ }^{*}\right.$ means $\left.\mathrm{p}<0.05\right)$. 


\section{Discussions}

Cancer patients are extremely prone to negative emotions, such as anxiety, depression and fear. If the psychological reactions of patients are too negative or patients fall into negative emotions for a long term, it would cause imbalance of hormone levels of patients' body, increase of secretion of adrenaline, and further bring about the decrease of immunity functions of their body and seriously hinder the comprehensive rehabilitation of patients. Therefore, it is very necessary to implement the clinical psychological care project.

PDRQ-15 refers to the doctor-patient relationship scale, and its total scores are the sum of scores of each item, and dimension scores are the sum of scores of each item in each dimension and the higher the scores are, the better the doctor-patient relationship becomes, vice versa. The PCL-C scale is used for preliminary screen of posttraumatic stress disorder, and the total scores can be obtained after rating and summary of 17 items and can show the degree of PTSD. The SAS-self-rating anxiety scale takes 50 scores as a boundary, if a patient obtains over 50 scores in the scale, he (she) would be judged as anxious. The SD-self-rating depression scale takes 52 scores as a boundary, if a patient obtains over 52 scores, he (she) would be judged as depressed. Research results showed that the total scores of CL-C, SAS, SDS and PDRQ-15 scales of cancer patients and their families after clinical psychological care intervention were significantly lower than those of cancer patients and their families before intervention and those in the control group, which indicated that psychological intervention can bring effective effects to cancer patients and their families.

The Pearson correlation coefficient is a linear correlation coefficient substantially in statistical methods, and the bigger the absolute value of the correlation coefficient is, the stronger the correlation becomes: if the correlation coefficient is closer to 1 or -1 , the correlation is stronger, if the correlation coefficient is closer to 0 , the correlation is weaker [10]. The results of Pearson correlation analysis of PCL-C, SAS and SDS with PDRQ-15 of cancer patients and their families indicated that PCL-C, SAS and SDS were strongly correlated with PDRQ-15.

Both of DA and NE have the functions of hormones and neurotransmitters, and are closely related to sympathetic activities and are thus frequently used as one of the indicators to measure sympathetic activities [11]. Through the determination of the secretion volume of DA and NE of cancer patients with PTSD and those without PTSD, it was discovered that the DA and NE levels of cancer patients with PTSD were all higher than those of cancer patients without PTSD, suggesting that the sympathetic activities of cancer patients under stress conditions may be further increased.

\section{Outcomes}

After psychological intervention by cancer patients and their families, anxiety, depression and doctor-patient relationship have improved, allowing patients to respond to diseases in a positive way, which is conducive to improving patients' 
clinical symptoms, improving patients' quality of life, and improving doctors and patients. Relationship satisfaction

\section{Conclusion}

In conclusion, the post-traumatic stress disorder, anxiety, depression and doctor-patient relationship of cancer patients and their families after psychological intervention were all improved. Such intervention helps patients respond to diseases in a positive manner, is favorable to improve the clinical symptoms of patients, strengthen the life quality of patients and enhance the satisfaction of doctor-patient relationship.

\section{Source of Paper Project Funding}

Hainan Natural Science Foundation, number: 818MS172; Funded by the National Natural Science Foundation of China, No. 81760255.

\section{Conflicts of Interest}

The authors declare no conflicts of interest regarding the publication of this paper.

\section{References}

[1] Wu, F. (2016) Current Situation and Outlook of Psychological Nursing of Cancer Patients. World Latest Medicine Information, 16, 33-35.

[2] Hu, S.P. and Du, C. (2013) Effect of Negative Emotions of Accompanying Persons on Cancer Patients and Intervention Adjustment. Journal of Qiqihar Medical College, 34, 306-307.

[3] Ren, H., Ou, J.F. and Ding, X.M. (2014) Linear Regression Analysis of Premorbid Life Events of Patients with Malignant Tumor. Medical Journal of National Defending Forces in Northwest China, 35, 26-28.

[4] Tao, X.H., Li, Z., Tian, F.Q., et al. (2017) The Effect of Comprehensive Psychological Nursing Intervention on Young Patients with Breast Cancer. Journal of International Psychiatry, 2, 2-7.

[5] Chai, J.W. and Wang, X. (2015) Cause Analysis and Prevention of Conflicts between Surgical Nurses and Families of Cancer Patients. Public Medical Forum Magazine, 19, 1685.

[6] Fan, C.N. (2015) Investigation and Analysis of Health Education Demands of Families of Patients with Cancer during Chemotherapy. Journal of Binzhou Medical University, 38, 311-312.

[7] Wang, H., Zhu, Y.C., Xu, J., et al. (2015) Research on Factors Related to Psychological Health and Psychological Intervention Needs of Convalescent Cancer Patients. The Journal of Medical Theory and Practice, 28, 2730-2732.

[8] Wu, Q.Y., Ye, Z.X. and Li, L. (2015) Measurement and Influencing Factors of Fear of Cancer Recurrence among Cancer Patients. Chinese Nursing Management, 15, 1020-1021.

[9] Wang, L. (2015) Psychological Intervention for Cancer Patients at Different Stages. World Latest Medicine Information, 15, 201-202. 
[10] Zhang, J.Y., Gao, R., Hu, J., et al. (2014) Comparison of Application of Grey Correlation Degree and Pearson Correlation Coefficient. Journal of Chifeng University (Natural Science Edition), 21, 1-2.

[11] Keane, W.F. and Lyle, P.A. (2003) Recent Advances in Management of Type 2 Diabetes and Nephropathy Lessons from the RENAAL Study. American Journal of Kidney Diseases, 41, 22-25. https://doi.org/10.1053/ajkd.2003.50078 